Supporting Information

\title{
Photothermal and Heat-Transfer Properties of Aqueous Detonation Nanodiamonds by Photothermal Microscopy and Transient Spectroscopy
}

Mikhail A. Proskurnin, * Liliya O. Usoltseva, Dmitry S. Volkov, Dmitry A. Nedosekin, Mikhail V. Korobov, and Vladimir P. Zharov

Prof. M. A. Proskurnin, Mrs. L. O. Usoltseva, Dr. D. S. Volkov, and Prof. M. V. Korobov, Chemistry Department, Lomonosov Moscow State University, Moscow, 119991, Russia Dr. D. A. Nedosekin and Prof. V. P. Zharov, Philips Classic Laser Laboratories, University of Arkansas for Medical Sciences, Little Rock, Arkansas, 72205, USA

* To whom correspondence should be addressed.E-mail:proskurnin@gmail.com SECTION S1. MANUFACTURER'S INFORMATION ON RDDM AND RUDDM NANODIAMONDS .1

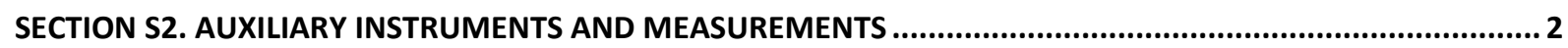

SECTION S3. THERMOPHYSICAL PROPERTIES OF NANODIAMOND AQUEOUS DISPERSIONS.............................

SECTION S4. WIDE-BEAM BACK-SYNCHRONIZATION THERMAL-LENS SPECTROMETER.................................. 7

SECTION S5. TRANSIENT THERMAL-LENS MEASUREMENTS................................................................. 10

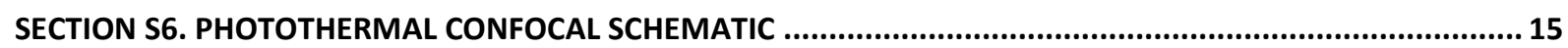

Section S1. Manufacturer's information on RDDM and RUDDM nanodiamonds

RUDDM 0-150

Specific surface (BET), $\mathrm{m}^{2} / \mathrm{g}: 320 \div 450$ depending on the cluster size. Solid impurities, $\%$ by mass. $\quad 0.5 \div 0.7$. Size, $50-70 \mathrm{~nm}$ Maximum limit, $250 \mathrm{~nm}$. $\underline{\text { https://real- }}$ dz.com/en/katalog/abrazivnyie-materialyi/nanoalmazyi-ruddm/ 
RDDM 0-0.25

Specific area $(\mathrm{BET}), \mathrm{m}^{2} / \mathrm{g}: 20 \div 25$. Solid impurities, $\%$ by mass: $0.1 \div 0.2$ Minimum Size $50-100$ nm; average size 0.15-0.20, Maximum limit, $0.39 \mu \mathrm{m}$. https://realdz.com/en/katalog/abrazivnyie-materialyi/polikristallicheskie-almaznyie-poroshki-rddm/

\section{Section S2. Auxiliary instruments and measurements}

DCS-30 TA Mettler instrument was used to measure specific heat from 288 to $323 \mathrm{~K}$. The scanning rate was $10 \mathrm{~K} / \mathrm{min}$. Each DSC sample weighed 20-30 mg. Preparation of ND samples for DSC: a portion of a ND sample was mixed with distilled water in a $1: 2$ ratio to give a gel sample (ND and water). The minimum amount of ND material necessary for one run was $1 \mathrm{mg}$. The DSC procedure is described in detail elsewhere. ${ }^{1}$

A Kern 770 analytical balance (Germany) was used for dry powder samples weighting. An Ecros 6500 shaker (Russia) was used for the preparation of dispersions. A GRAD 28-35 ultrasound bath (USB) from Grad-Technology (Russia) was used for preparing ND dispersions in water. A SNOL 20/300 heating oven (Snol-Term Ltd., Russia) was used for the evaporation of ND aqueous dispersions. Automatic Eppendorf Research pipettes (Eppendorf International, Germany) were used for the preparation of calibration solutions. Polypropylene test tubes (Axygen, USA) were used for solution preparation and storage.

A microfluidic slit rheometer, microVISC (Rheosense Inc., CA) was employed to measure the steady shear viscosity of the solutions. The specific microfluidic device used in this study, microVISC Type A chipset (13HA05100058), consists of a rectangular slit formed with glass and Si pressure sensor array. The pressure drop, $\Delta P$, required to drive the flow with rate, $\mathrm{Q}$, is related to the wall shear stress, $\tau$, by the expression $\Delta P=2 L(w+h) \tau$ ( $L$ is sensor position). The nominal wall shear rate, $\gamma$, associated with fully developed laminar flow of a Newtonian fluid is $\gamma=6 Q / w h^{2}$. In a typical experiment, the flow rate, $\mathrm{Q}$, is varied using a syringe pump and a $400 \mu \mathrm{L}$ disposable pipet (Rheosense Inc., CA). The microVISC device records the pressure drop as a function of flow rate, and the data is then used to determine the nominal or apparent viscosity $\eta(\gamma)=\tau / \gamma$. The fluid temperature throughout the instrument was maintained at desired constant values using thermal jackets. Water was used as reference liquid to ensure the accuracy of the rheometer before each measurement. At least duplicated samples were measured, and average viscosity values based on 3-5 measurements were recorded. 
Densities were determined with VIP-2MP (Russia) vibrating-tube densimeter. The following expression was used to obtain densities of the solutions: $\rho=A+B \tau^{2}$, where $\tau$ is the oscillation period and $A$ and $B$ are coefficients, determined from calibration at the $298.15 \mathrm{~K}$ by known densities and oscillation periods of ambient air, ultrapure water and standard material - REP$12\left(1090.32 \mathrm{~kg} \cdot \mathrm{m}^{-3}\right.$, produced and certificated by D. I. Mendeleev Institute for Metrology, Russia, St. Petersburg). The temperature was maintained by built-in thermostat; the standard uncertainty of registered temperature was $0.02^{\circ} \mathrm{C}$. The standard deviation for measurement of solution density is $0.1 \mathrm{~kg} \cdot \mathrm{m}^{-3}$.

\section{Section S3. Thermophysical properties of nanodiamond aqueous dispersions}

\section{S3.1. Density of nanodiamond dispersions}

The density dependence on concentration of various nanodiamond brands is shown in Figure S1. Linear dependences for all the studied brands we found, and they are slightly dependent on the brand.

The equation for the density of two-phase mixtures for particles of micrometer size is shown previously for slurry flows ${ }^{2}$. Pak and Cho ${ }^{3}$ introduced the same equation for the density $\rho_{n f}$ of nanofluids with nanometer-size particles:

$$
\rho_{n f}=\phi \rho_{\mathrm{p}}+(1-\phi) \rho_{s}
$$

where $\phi$ is ND volume fraction; $\rho_{\mathrm{p}}$ is particle density and $\rho_{s}$ is neat solvent density. For nanofluids containing inorganic particles $\left(\mathrm{Al}_{2} \mathrm{O}_{3}, \mathrm{Sb}_{2} \mathrm{O}_{5}: \mathrm{SnO}_{2}, \mathrm{ZnO}\right)$ in 60:40 ethylene glycol/water, good agreement was obtained with this theoretical equation Eq. (S1), but the deviation increased with particle v/v concentration. ${ }^{4}$

This equation was used for estimation of density for aqueous, ${ }^{5}$ mixed ${ }^{6}$ and organic ${ }^{7}$ nanofluids of nanodiamond. By so doing, densities of particles are needed as initially ND w/v concentrations are used in experiments. As for nanodiamond powders, manufacturers often do not provide this parameter. Samples densities were determined by a hydrostatic weighing in distilled water ${ }^{8}$ or by pycnometry (helium). ${ }^{9-10}$

Thus, we estimated nanodiamond densities from the linear dependence:

$$
\rho_{n f}=\rho_{n d} c / \rho_{n d}+\left(1-c / \rho_{n d}\right) \rho_{w}
$$


with a slope $\left(1-\rho_{w} / \rho_{n d}\right)$. Estimated values for $50 \mathrm{mg} / \mathrm{mL}$ are presented in Table $\mathrm{S} 1$, according to NIST, diamond density is $3.515 \mathrm{~g} / \mathrm{mL}$. The estimation of ND density from the linear equations result in approximation values of 2.9-3.6, which is close to pycnometric density of nanodiamond powders of 3.1-3.2. ${ }^{11-12}$

Table S1. Densities of aqueous dispersions of ND for $50 \mathrm{mg} / \mathrm{mL}(n=3 ; P=0.95)$

\begin{tabular}{|l|l|l|l|l|}
\hline ND & Slope of Eq. $(\mathrm{S} 2) \times 10^{4}$ & $\mathrm{R}^{2}$ & $\rho_{n f} \mathrm{~g} / \mathrm{mL}$ (increase, \%) & $\rho_{\text {nd, }} \mathrm{g} / \mathrm{mL}$ \\
\hline RUDDM & $6.51 \pm 0.02$ & 0.99999 & $1.029 \pm 0.003(3.3)$ & $2.86 \pm 0.01$ \\
RDDM & $7.10 \pm 0.01$ & 0.99999 & $1.032 \pm 0.002(3.6)$ & $3.44 \pm 0.01$ \\
SDND & $7.21 \pm 0.03$ & 0.99997 & $1.033 \pm 0.003(3.6)$ & $3.57 \pm 0.01$ \\
\hline
\end{tabular}

$\mathrm{Yu}$, et al. ${ }^{13}$ constructed a settling time dependence of the density of the nanofluid prepared with $0.15 \% \mathrm{v} / \mathrm{v}$ addition of plasma-treated and untreated diamond nanoparticles in water. Also, they demonstrate that with $0.15 \% \mathrm{v} / \mathrm{v}$ addition of plasma treated nanoparticles into water, a 5-10\% increase in both fluid density and viscosity was observed. ${ }^{13}$

\section{S3.2. Specific heat of nanodiamond dispersions}

DSC is most used for measuring specific heats of nanofluids. ${ }^{14}$ Nanodiamond brand does not significantly influences on specific heat of nanofluid (Figure S2, a). For all the brands, the specific heat of nanodiamonds shows a rather distinct drop upon a low concentration of NDs

The specific heat for a ND nanofluid as a function of concentration regardless of the brand in the concentration range $0-280 \mathrm{mg} / \mathrm{mL}$ can be described as a linear equation:

$$
C_{p, n f}=(4.16 \pm 0.03)-(3.3 \pm 0.2) \cdot 10^{-3} c
$$

with the goodness of fit $R^{2}=0.98$. However, the estimation of nanodiamond phase specific heat is not possible as the curve for the whole range of volume fractions of ND should have a sigmoidal form with a plateau at low and high concentrations of nanodiamonds (small changes compared to pure phases). 
Pak and Cho ${ }^{3}$ and Xuan and Roetzel ${ }^{15}$ proposed two expressions to determine the specific heat of nanofluids based on nanofluid volume fraction, $\phi$ :

$$
\begin{aligned}
& C_{\mathrm{p}, \mathrm{nf}}=\phi C_{\mathrm{p}, \mathrm{ND}}+(1-\phi) C_{\mathrm{p}, \mathrm{f}} \text { Model I (S4) } \\
& \left(\rho C_{\mathrm{p}}\right)_{\mathrm{nf}}=\phi\left(\rho C_{\mathrm{p}}\right)_{\mathrm{ND}}+(1-\phi)\left(\rho C_{\mathrm{p}}\right)_{\mathrm{f}} \text { Model II (S5) }
\end{aligned}
$$

Ghazvini, et al. ${ }^{16}$ presented a correlation of specific heat of nanofluid with $1 \%$ weight fraction of nanodiamond in engine oil as a function of temperature. Model I was previously used for the estimation of nanofluid specific heat for aqueous nanodiamond dispersion. ${ }^{5}$

In our opinion, correlation equations based on volume fractions of components, though simpler from the experimental point of view, are not correct as heat capacity is a thermodynamic parameter depending on the molar fractions of components; thus, equations based on mass or volume fractions should be nonlinear by definition. Thus, a more correct equation should deal with molar heat capacities and molar fractions of ND and water (see Figure S2, b)

$$
C_{\mathrm{P}, \mathrm{M}, \mathrm{nf}}=v C_{\mathrm{P}, \mathrm{ND}} M_{\mathrm{ND}}+(1-v) C_{\mathrm{P}, \mathrm{water}} M_{\mathrm{water}},
$$

where $v$ is ND molar fraction. Using this equation, one can obtain the mean molar heat capacity of nanofluid, which can be recalculated to the heat capacity of nanofluids through the averaged molar mass of the fluid taking into account the molar masses of components $M_{\mathrm{ND}}$ and $M_{\text {water }}$. To calculate specific heat of nanodiamonds, the estimated values of nanodiamond density (Table S1) were used.

Table S2. Specific heat of nanodiamonds

\begin{tabular}{|l|l|}
\hline Material & $C_{P}, \mathrm{~J} / \mathrm{g} / \mathrm{K}$ \\
\hline RDDM (this study) & $0.50 \pm 0.03$ \\
RUDDM (this study) & $0.75 \pm 0.04$ \\
Diamond $^{17 *}$ & 0.51 \\
Graphite $^{18 * *}$ & $0.67-0.75$ \\
${\text { Impurity free ND, calc. }{ }^{19}}^{\text {ND (Sinta, Belarus and Altay SPA, Russia) brands }{ }^{19}}$ & 0.57 \\
\hline
\end{tabular}

\footnotetext{
* According to NIST Webbook https://webbook.nist.gov/cgi/cbook.cgi?ID=C7782403\&Mask=2

** According to NIST Webbook https://webbook.nist.gov/cgi/cbook.cgi?ID=C7782425\&Mask=2
} 
Specific heat of RUDDM, RDDM and SDND from Eq. (S6) gives 0.75, 0.45, and $0.56 \mathrm{~J} / \mathrm{g} / \mathrm{K}$, respectively (linear dependences to $v=0.23$ ). These values are well-correlated with experimental powder measurements (Table S2) and are close to the value of the heat capacity for the impurity-free ND and other ND brands measured as powders $(0.57$ and $0.65 \mathrm{~J} / \mathrm{g} / \mathrm{K}) .{ }^{19}$ Certainly, a detailed study of specific heat relationship on the concentration was out of the scope of this study, and we were limited to the concentration range with expected effect on thermal conductivity as nanofluids. For the real dependence, the whole dependence should be build including ND pastes, gels, and concentrated sols. The recent paper ${ }^{20}$ shows serious changes for water at very low ND concentrations due to the structure-forming effect.

\section{S3.3 Dynamic viscosity of nanodiamond dispersions}

Viscosities of aqueous dispersions are depicted in Figure S3. Three types of ND capable of forming concentrated solutions were chosen for viscosity measurement. Concentration dependences differ, but, as for RUDDM and RDDM curves, they are similar to data by Vul, et al. ${ }^{21}$ When the concentration exceeds 50 and $90 \mathrm{mg} / \mathrm{mL}$ for RUDDM and RDDM respectively, the viscosity begins to deviate from linearity. According to Sundar, et al., ${ }^{22}$ available theoretical formulas for estimation viscosity include particle volume concentration to the different power. The concentration dependence for SDND is polynomial of degree 3 or higher. Since viscosity plays a big role in systems involved in fluid flow, these dependences could be used in selecting ND suspension with the best thermal performance. 


\section{Section S4. Wide-beam back-synchronization thermal-lens spectrometer}

\section{S4.1 Back-synchronization thermal lensing principle}

In the back-synchronization mode of thermal lensing each heating-cooling measurement cycle is different from a common synchronous (lock-in) mode. The durations of the heating and cooling half-cycles do not coincide, and each half-cycle begins only after a reliably detected steady state (defined as not exceeding the maximum permissible relative standard deviation of the convergence of the intensity of the probe beam at the detector plane).

These steady states correspond to the achievement of the following equilibria. In the heating half-cycle, to the thermal equilibrium in the sample upon the formation of a stationary thermal lens; in the cooling half-cycle, to the complete dissipation of the thermal field of the thermal lens at the end of heating. Thus, at each stage (the blooming and dissipation of a thermal field, a stationary thermal lens, and probe-intensity acquisition in the absence of a thermal lens), different measurement modes are defined on the thermal properties and concentration of the sample in question.

As a result, the conditions of thermal-lens measurements are determined by the reliable achievement of a stationary state and signal accumulation for this state. In the lock-in mode of thermal lens measurements, incomplete dissipation of heat at the end of the measurement cycle is common ${ }^{23}$ and, as a result, a decrease in the sensitivity of thermal lens measurements due to smaller changes in temperature during one cycle of interruption or on/off of the excitation laser. The back-synchronization mode provides the maximum temperature change in one heatingcooling cycle of the sample due to the complete dissipation of the absorbed laser radiation at the end of the cycle and, thus, increases the measurement sensitivity and accuracy.

Other advantages of this modality are $(i)$ the possibility of detection under batch and flow conditions with no change in the optical-scheme design of the instrument; (ii) the possibility to switch between transient and steady-state measurements within a single set of experiments; and (iii) a wide linear dynamic range (more than five orders of magnitude of detectable absorbances) including strongly absorbing and scattering samples.

\section{S4.2 Spectrometer}

The spectrometer was developed ${ }^{24}$ and optimized for the determination of solutions with a light absorption down to $1 \times 10^{-6}$ abs. units. The scheme is given at Figure S4. The key parameters are summarized in Table S3 (from this point on, the subscript " $p$ " will denote the probe beam, and 
the subscript " $e$ " will stand for the excitation beam). The uncertainty in the determination of the excitation beam radius was calculated using a reference sample (a standard solution of ferroin with the concentration of $1.1 \mu \mathrm{M}$ ) with known thermal diffusivity and absorbance (from spectrophotometry). The optimization of the thermal lens spectrometer geometry to account for convection processes or the Soret effect was out of the scope of this work and is described elsewhere ${ }^{24}$. Quartz cells with optical path length of $10 \mathrm{~mm}$ were used throughout this work.

Table S3. Parameters of the wide-beam thermal-lens spectrometer

\begin{tabular}{lll}
\hline Excitation $\mathrm{Ar}^{+}$laser & Wavelength, $\lambda_{e}$ & $514.5 \mathrm{~nm}$ \\
& Max power (TEM 00 mode), $P_{e}$ & $210 \mathrm{~mW}$ \\
Innova 90-6 (Coherent, USA) & Waist, $\omega_{0 \mathrm{e}}$ & $59.8 \mu \mathrm{m}$ \\
\hline Probe He-Ne laser HRP020 & Wavelength, $\lambda_{p}$ & $632.8 \mathrm{~nm}$ \\
(ThorLabs, USA) & Power (TEM 00 mode), $P_{p}$ & $2 \mathrm{~mW}$ \\
\hline Chopper repetition rate & $\varphi, \mathrm{Hz}$ & $0.1-100$
\end{tabular}

\section{S4.3. Steady-state thermal-lens data treatment}

Thermal lens signal is determined by a relative change of the probe laser at the detection plane, Eq. (2) and is described as 23

$\vartheta(t)=P_{e} B(t) \cdot E_{0} \cdot \varkappa l$

where $P_{\mathrm{e}}$ is the excitation laser power, $\omega_{0 \mathrm{e}}$ is the excitation beam waist radius, $\mathcal{x}=2.303 \varepsilon \mathrm{C}$ is the linear light-absorption coefficient of the sample, $l$ is sample path length, $\varepsilon$ is the molar absorptivity, and $c$ is molar concentration of the absorbing substance in the sample. The factor $E_{0}$ is the thermooptical constant $\left(E=P_{e} E_{0}\right)$, characterizing the strength of the thermal-lens effect per a unit excitation (pump) laser power:

$E_{0}=(-d n / d T) / \lambda_{p} k$

Here, $k$ is the thermal conductivity, $\lambda_{p}$ is the probe laser wavelength, and the temperature gradient of refractive index $(d n / d T) \propto \alpha_{T}$ (volumetric thermal expansion coefficient).

$B(t)$ is the time-dependent geometrical constant of the optical schematic ${ }^{25-26}$

$B(t)=1 / 2 \tan ^{-1}\left[\frac{2 m V}{\left\{\left[(1+2 m)^{2}+V^{2}\right]\left(t_{c} / 2 t\right)+1+2 m+V^{2}\right\}}\right]=1 / 2 \tan ^{-1}\left[\frac{a}{\left(b t_{c} / 2 t+c\right)}\right]$. 
Here, $m$ is the ratio of cross-section areas of the probe and excitation beams at the sample, and $V$ is the relative distance from the excitation waist to the sample, a traditional notation of TLS geometry parameters proposed by R. Snook, J. Shen and M. Baesso ${ }^{25-26}$ and $a, b$, and $c$ are related geometry constants of the spectrometer used in the approximation for the equation simplicity's sake. ${ }^{27}$

The steady-state cw thermal-lens signal is

$\vartheta(\infty)=\left(I_{p}(0)-I_{p}(\infty)\right) / I_{p}(\infty) \quad(\mathrm{S} 10)$

and is defined as $25-26$

$\vartheta(\infty)=B P_{e} E_{0} \gamma l=B E \cdot 2.303 \varepsilon l c=B \cdot \theta(\mathrm{S} 11)$

Here, $\theta \equiv 2.303 E_{0} P_{e} \varepsilon l c$ is the steady-state thermal-lens signal corrected for the steady-state geometry constant $B(t \rightarrow \infty)$.

The experimental values of sample absorbance $A_{\exp }$ were corrected to light scattering,

$$
A=A_{\text {exp }}-A_{\mathrm{s}}
$$

The experimental values of the wide-beam PT signal $\theta$ were also corrected to take into account a decrease in the excitation power due to light-scattering losses $A_{\mathrm{s}}$ in solutions:

$$
\theta_{\text {corr }}=\theta\left(A+A_{s}\right) / A
$$

where $A$ is sample absorbance.

The recalculation of the absorbance from photothermal measurements $\left(A_{\mathrm{PT}}\right)$ were calculated from the equation deduced from (S11) and the Beer's law $A=\varepsilon l c$

$$
A_{P T}=\theta_{\text {corr }} / 2.303 E_{0} P_{e}
$$

For calculations of characteristic data for dispersed systems, we used the data given in ${ }^{23}$ as well as previously developed theoretical description for signal generation in thermal-lens spectrometry for multipoint-absorbing (disperse) solutions, ${ }^{28}$ see Section S5. 


\section{Section S5. Transient thermal-lens measurements}

\section{S5.1 Principle}

Thermal lens spectrometry is a photothermal method, i.e. the signal depends on thermophysical characteristics of the medium, primarily thermal diffusivity. Thermal-lens measurements is based on the establishment of thermal equilibrium in the medium under the action of laser radiation, and for it there are not only equilibrium (stationary), but also transient signals that depend on different parameters.

TLS is often used to establish the thermophysical parameters of a sample, since the type of socalled time-resolved curves, which are described by the equation in the framework of the theory of thermal lens effect for true solutions. Such a dynamic thermal lens curve consists of several sections (Fig. S5): at the initial instant of time, the laser beam exciting the thermal lens does not enter the sample; after the shutter (chopper) is opened, the formation of a temperature field begins, which acts as a concave lens (thermal lens effect). Next, the stationary thermal lens effect signal is recorded, and the measurement is carried out until the standard deviation of the recorded signal begins to be lower than the established criterion of measurement accuracy. Next, the chopper again blocks the beam of the excitation laser, and the dissipation of the thermooptical element occurs; then recording of the next measurement cycle begins. The development curve is determined by the thermal diffusivity during heating and thermal conductivity (effusivity) during cooling. ${ }^{23,29}$

In homogeneous systems, the inducing beam heats part of the solution, after which heat propagates from the center of the beam to the periphery (Fig. S6, a-c). The maximum temperature is observed in the center of the irradiation region of the object by the excitation beam, and as the distance from this region decreases, the temperature decreases, while the radial temperature profile corresponds to the energy distribution in the incident beam. The time to establish thermal equilibrium is determined by the thermal diffusivity of the medium.

The heat transfer process in dispersed systems differs from the case of true solutions. At different time intervals, different components of the system are responsible for heat transfer. ${ }^{27}$ When a heterogeneous sample consisting of light-absorbing nanoparticles and a non-absorbing dispersion medium is irradiated by inducing radiation, only the former absorb the inducing radiation, and in the initial period of time the thermal diffusivity of the dispersed phase contributes to heat distribution (Fig. S6, d). First, each particle forms a local thermal profile, a nanolens ${ }^{30-31}$ (Fig. S6, e). Then the heated zones merge, a single radial temperature profile 
(thermooptical element) is formed, ${ }^{28,32}$ which is determined by the spatial distribution of intensity in the excitation beam, and the system comes into a state of thermal equilibrium (Fig. S6, f). However, due to the fact that at different stages of the development of the thermal lens effect, heat distribution is determined by the contributions from the dispersed particles and the thermophysical parameters of the dispersion medium, the form of time-resolved thermal lens signals changes in comparison with true solutions with the same absorbance. ${ }^{27}$ A similar behavior is characteristic of aqueous systems of metal nanoparticles and proteins.

\section{S5.2 Transient data treatment}

The data of 50-100 replicate transient thermal lens curves (S7) were measured and averaged. The averaged curves were approximated as ${ }^{25-26}$

$$
I(t) / I(0)=\left(1-1 / 2 \theta \tan ^{-1}\left[a /\left(b t_{c} / 2\left(t-t_{0}\right)+c\right)\right]\right)^{2},
$$

where $t_{0}$ is the delay time, which depends on transfer open/close times of the mechanical shutter, which is found empirically from the real start of the change of the probe-beam intensity. The shape of the thermal-lens curve for nanodiamonds is presented in Figure S7. From the viewpoint of non-equilibrium (time-dependent) processes with characteristic time differing from the neat solvent, Eq. (S15) was solved for the effective, time-dependent characteristic time $\tilde{t}_{c}(t):$

$\left.\left.\tilde{t}_{c}(t)=2 \cot [X(t)](a-c \cdot \tan [X(t)])\left(t-t_{0}\right)\right]\right) / b$,

where the auxiliary function $X(t) \equiv 2(1-\sqrt{I(t) / I(0)}) / \theta$. The effective thermal diffusivity as a function of time, $\widetilde{D}_{T}(t)$, was calculated from (S16) as

$\widetilde{D}_{T}(t)=\omega_{0 e}^{2} / 4 \tilde{t}_{c}(t)$

$\tilde{t}_{c}(t)$ curves show two types of periodic noises, a short-time periodic noise due to fluctuations of the probe-laser beam intensity and periodic beatings with a frequency of $15 \mathrm{~Hz}$ caused by the vibrations imposed on chopper opening/closing, which become visible on $\tilde{t}_{c}(t)$, especially for concentrated dispersions. Thus, the calculated curves $\tilde{t}_{c}(t)$ and $\widetilde{D}_{T}(t)$ were subjected to two-stage smoothing: first weighted adjacent averaging (5 points) to remove short-scale fluctuations followed by a Savitzky-Golay smoothing to remove the periodic noise. This twostage algorithm provided preserving the general shape of the curve but affected the starting point and the maximum value of the curve. 


\section{S5.3. Thermophysical parameters of aqueous nanodiamond dispersions}

Thermal diffusivity was calculated from Eqs. (1) and (S16). The volumetric heat capacity $\rho C_{p}$ was calculated from the experimentally determined density and isobaric specific heat data.

The thermal conductivity $k$ and thermal effusivity $e_{T}$ were calculated from the experimental thermal diffusivity and from the specific heat capacity and density of the analyzed sample

$k=\rho C_{p} D_{T} \quad(\mathrm{~S} 18)$

$e_{T}=\sqrt{k \rho C_{p}}=\rho C_{p} \sqrt{D_{T}}$

When apparent time-dependent values $\widetilde{D}_{T}(t)$, Eq. (S17), were used, thus producing time curves of apparent thermal conductivity and apparent thermal effusivity, $\tilde{k}(t)$ and $\tilde{e}_{T}(t)$, respectively.

Thermal diffusion length $\mu_{T}$ during time-resolved and microscopic measurements at the moment $t$ was calculated as

$\mu_{T}(t)=2 \sqrt{D_{T} t}$

For disperse systems, apparent time-dependent values $\widetilde{D}_{T}(t)$, Eq. (S17), was used instead.

The experimental thermooptical constant, eq. (S8) was determined from the thermal-lens data for samples with known absorbance $A$ (from spectrophotometric measurements):

$E_{0}=\frac{\theta}{2.303 P_{e} A}$

The value of $(d n / d T)$ from the steady-state thermal-lens data was estimated for the probe wavelength of $632.8 \mathrm{~nm}$ from eq. (S8) and the value of thermal conductivity from (S18).

For calculations of characteristic data for dispersed systems, we used the data given in ${ }^{23}$ as well as calculations from the previously developed theoretical description for signal generation in thermal-lens spectrometry for multipoint-absorbing (disperse) solutions. ${ }^{28}$ The thermal expansion coefficient was estimated from the thermal-lens value of $(d n / d T)$ and the extrapolation equation

$\frac{d n}{d T} \approx\left(\frac{\partial n}{\partial T}\right)_{p}=-\alpha_{T} \frac{\left(n^{2}-1\right)\left(n^{2}+2\right)}{6 n}$ 
deduced from the Lorentz-Lorenz relation according to the approach described. ${ }^{33-35}$

\section{S5.4 Modelling of multipoint secondary heating}

Also, for estimating the thermal field, we used previously developed numerical description for signal generation in photothermal lensing for multipoint-absorbing (disperse) solutions. ${ }^{28,36}$

This approach is based on the same calculation of the signal from the phase shift, but the temperature change $\Delta T(r, z, t)$ is found by a summation of individual thermal waves from multiple heat sources distributed in $N$ thin layers plus the conventional contribution from the light-absorbing background with a due account for the random movement (shift) of the heat sources in the signal-generation process).

$\Delta T(z, r, t)=\sum_{n=1}^{M} \sum_{i=1}^{N} \Delta T_{n, i}(z, r, t)+\sum_{n=1}^{M} \sum_{i=1}^{N} \Delta T_{n, i}(z+Z, r+R, t)+\ldots$

Here, $M$ is the number of excitation pulses in a train (cw excitation is simulated with a large train of ultrashort pulses). The second double-sum term represents the shifting of the heat element during the heating period by substituting the initial variables $(r, z)$ to $(r+R, z+Z)$, where $(R, Z)$ is the shift vector. Thus, summing the shifted heat functions, Eq. (S23), gives a picture of heat-generation in the system without binding it to the radial symmetry of the excitation beam. The model represents heat flow from every individual heat source; thus, resulting in the exact spatially resolved thermal picture, which is much closer to the real situation in the description of dilute solutions. The PT characteristics for heterogeneity are not considered in the calculation of the heat diffusion as the contribution of the total heat-source volume to the total sample volume is negligible, and the heating of heterogeneity is considered instantaneous, thus, only the PT properties of the medium are taken into account. The applications of a quasi-cw function for the description of the solution excitation do not prevent the calculation of transient functions of the temperature profile and PT signal.

The temperature distribution in the layer $i, \Delta T_{i}$ is based on a separate consideration of the axial (below, the term in curly brackets) and radial (the term in square brackets) components of the temperature profile:

$$
\begin{gathered}
\Delta T_{n, i}(r, z, t)=\frac{8 D_{T} t_{0}}{\sqrt{\pi} k}\left(\alpha_{i} I_{i-1}\right)\left\{e^{\left(\frac{r^{2}}{\omega_{0 e^{+4 D} t}^{2}}\right)}\left(\omega_{0 e}^{2}+4 D_{T} t\right)\right\} \times \\
\times\left[e^{\alpha_{i}\left(z+z_{i-1}\right)} e^{\alpha_{i}^{2} D_{T} t}\left\langle\operatorname{erfc}\left\{\frac{\left(z+z_{i-1}\right)}{2 \sqrt{D_{T} t}}+\alpha_{i} \sqrt{D_{T} t}\right\}-\operatorname{erfc}\left\{\frac{\left(z+z_{i}\right)}{2 \sqrt{D_{T} t}}+\alpha_{i} \sqrt{D_{T} t}\right\}\right)\right] .
\end{gathered}
$$


Here $I_{i-1}$ is the intensity of the excitation radiation incident to the layer $i$. The parameter $t_{0}$ is the quasi-pulse duration (expresses the energy transmitted to the absorbing medium only, while the transfer of the total pulse energy is considered instantaneous. ${ }^{32}$ This equation is used as is for pulsed excitation. The temperature response on cw irradiation is achieved by the summation of the temperature responses on a series of laser quasi-pulses, with each quasi-pulse starting at the moment $t_{n}=(n-1) t_{0}$, where $n$ is the ordinal of the quasi-pulse. Heat-dissipation functions from different heat sources form the final temperature profile. The heat-wave profiles generated by different heat sources are described with the same function and PT response amplitudes differ according to the particle position in the excitation laser beam. 


\section{Section S6. Photothermal confocal schematic}

\section{S6.1. Principle}

The signal curve on the distance from the probe-beam waist on the sample in confocal photothermal microscopy exhibits only one maximum (not two, as in conventional PT schematics corresponding to focusing/defocusing). Although confocal PTM uses the same detection scheme with a pinhole to measure probe beam modulation, and the pinhole position is carefully adjusted so that it lies on a plane one Rayleigh range away from the probe beam waist image plane (Figure S8, a). The excitation laser waist is carefully adjusted to match the waist pf the probe beam (mode-matched configuration); however, a possible adjustment mismatch or chromatic aberrations of the optical system only affect signal amplitude, not confocal detection. ${ }^{37-38}$ The instrument is described in the main text; the schematic is given in Figure S9.

\section{S6.2. Nonlinear mode}

Nonlinear effects accompanying fast heating of nanoparticles with a short (pico- or nanosecond) pulses are related to the formation of nano- or micrometer-sized bubbles around the heated particles. $^{38-39}$. In the nonlinear mode, local temperature increase exceeding the nanobubble formation threshold provided a sharp change in the probe beam intensity (Figure S8). The comparison of a nanodiamond sample in the nonlinear mode and a blank signal is shown in Figure S10. 


\section{References for the Supporting Information}

1. Korobov, M. V.; Batuk, M. M.; Avramenko, N. V.; Ivanova, N. I.; Rozhkova, N. N.; Ōsawa, E., Aggregate Structure of "Single-Nano Buckydiamond" in Gel and Dried Powder by Differential Scanning Calorimetry and Nitrogen Adsorption. Diamond Relat. Mater. 2010, 19, 665-671.

2. Cheremisinoff, N. P., Encyclopedia of Fluid Mechanics: Slurry Flow Technology; Gulf Publishing Company, Book Division, 1986.

3. Pak, B. C.; Cho, Y. I., Hydrodynamic and Heat Transfer Study of Dispersed Fluids with Submicron Metallic Oxide Particles. Experimental Heat Transfer an International Journal 1998, 11, 151-170.

4. Vajjha, R.; Das, D.; Mahagaonkar, B., Density Measurement of Different Nanofluids and Their Comparison with Theory. Pet. Sci. Technol. 2009, 27, 612-624.

5. Sundar, L. S.; Hortiguela, M. J.; Singh, M. K.; Sousa, A. C., Thermal Conductivity and Viscosity of Water Based Nanodiamond (Nd) Nanofluids: An Experimental Study. International Communications in Heat and Mass Transfer 2016, 76, 245-255.

6. Sundar, L. S.; Singh, M. K.; Sousa, A. C. M., Enhanced Thermal Properties of Nanodiamond Nanofluids. Chem. Phys. Lett. 2016, 644, 99-110.

7. Taha-Tijerina, J. J.; Narayanan, T. N.; Tiwary, C. S.; Lozano, K.; Chipara, M.; Ajayan, P. M., Nanodiamond-Based Thermal Fluids. ACS Appl Mater Interfaces 2014, 6, 4778-85.

8. Kidalov, S.; Shakhov, F.; Vul, A. Y., Thermal Conductivity of Nanocomposites Based on Diamonds and Nanodiamonds. Diamond Relat. Mater. 2007, 16, 2063-2066.

9. Larionova, I.; Frolov, A.; Poleva, L.; Bychin, N., Study of the Composition and Physicochemical Properties of Diamond Hydrogels. Colloid J. 2004, 66, 372-374.

10. Larionova, I.; Kuznetsov, V.; Frolov, A.; Shenderova, O.; Moseenkov, S.; Mazov, I., Properties of Individual Fractions of Detonation Nanodiamond. Diamond Relat. Mater. 2006, $15,1804-1808$.

11. Kuznetsov, V. L.; Butenko, Y. V., 13 - Diamond Phase Transitions at Nanoscale. In Ultrananocrystalline Diamond, Shenderova, O. A.; Gruen, D. M., Eds. William Andrew Publishing: Norwich, NY, 2006; pp 405-475.

12. Mansoori, G. A.; George, T. F.; Assoufid, L.; Zhang, G., Molecular Building Blocks for Nanotechnology: From Diamondoids to Nanoscale Materials and Applications; Springer New York, 2007.

13. Yu, Q.; Kim, Y. J.; Ma, H., Nanofluids with Plasma Treated Diamond Nanoparticles. Appl. Phys. Lett. 2008, 92, 103111.

14. Shahrul, I.; Mahbubul, I.; Khaleduzzaman, S.; Saidur, R.; Sabri, M., A Comparative Review on the Specific Heat of Nanofluids for Energy Perspective. Renewable and Sustainable Energy Reviews 2014, 38, 88-98. 
15. Xuan, Y.; Roetzel, W., Conceptions for Heat Transfer Correlation of Nanofluids. International Journal of heat and Mass transfer 2000, 43, 3701-3707.

16. Ghazvini, M.; Akhavan-Behabadi, M.; Rasouli, E.; Raisee, M., Heat Transfer Properties of Nanodiamond-Engine Oil Nanofluid in Laminar Flow. Heat Transfer Engineering 2012, 33, $525-532$.

17. Reeber, R. R.; Wang, K., Thermal Expansion, Molar Volume and Specific Heat of Diamond from 0 to 3000k. Journal of electronic materials 1996, 25, 63-67.

18. Lutcov, A. I.; Volga, V. I.; Dymov, B. K., Thermal Conductivity, Electric Resistivity and Specific Heat of Dense Graphites. Carbon 1970, 8, 753-760.

19. Vasiliev, O. O.; Muratov, V. B.; Kulikov, L. M.; Garbuz, V. V.; Duda, T. I., Special Features of the Heat Capacity of Detonation Nanocrystalline Diamond. Journal of Superhard Materials 2015, 37, 388-393.

20. Batsanov, S. S.; Dan'kin, D. A.; Gavrilkin, S. M.; Druzhinina, A. I.; Batsanov, A. S., Structural Changes in Colloid Solutions of Nanodiamond. New Journal of Chemistry 2020, 44, 1640-1647.

21. Vul, A. Y.; Eydelman, E.; Inakuma, M.; Ōsawa, E., Correlation between Viscosity and Absorption of Electromagnetic Waves in an Aqueous Uncd Suspension. Diamond Relat. Mater. 2007, 16, 2023-2028.

22. Sundar, L. S.; Sharma, K.; Naik, M.; Singh, M. K., Empirical and Theoretical Correlations on Viscosity of Nanofluids: A Review. Renewable and Sustainable Energy Reviews 2013, 25, 670-686.

23. Bialkowski, S. E.; Astrath, N. G. C.; Proskurnin, M. A., Photothermal Spectroscopy Methods; Wiley, 2019, p 512.

24. Proskurnin, M. A.; Zhidkova, T. V.; Volkov, D. S.; Sarimollaoglu, M.; Galanzha, E. I.; Mock, D.; Nedosekin, D. A.; Zharov, V. P., In Vivo Multispectral Photoacoustic and Photothermal Flow Cytometry with Multicolor Dyes: A Potential for Real-Time Assessment of Circulation, Dye-Cell Interaction, and Blood Volume. Cytometry A 2011, 79, 834-47.

25. Shen, J.; Lowe, R. D.; Snook, R. D., A Model for Cw Laser-Induced Mode-Mismatched Dual-Beam Thermal Lens Spectrometry. Chem. Phys. 1992, 165, 385-396.

26. Baesso, M. L.; Shen, J.; Snook, R. D., Time-Resolved Thermal Lens Measurement of Thermal-Diffusivity of Soda Lime Glass. Chem. Phys. Lett. 1992, 197, 255-258.

27. Mikheev, I. V.; Usoltseva, L. O.; Ivshukov, D. A.; Volkov, D. S.; Korobov, M. V.; Proskurnin, M. A., Approach to the Assessment of Size-Dependent Thermal Properties of Disperse Solutions: Time-Resolved Photothermal Lensing of Aqueous Pristine Fullerenes C60and C70. J. Phys. Chem. C 2016, 120, 28270-28287.

28. Brusnichkin, A. V.; Nedosekin, D. A.; Proskurnin, M. A.; Zharov, V. P., Photothermal Lens Detection of Gold Nanoparticles: Theory and Experiments. Appl. Spectrosc. 2007, 61, 1191-201. 
29. Proskurnin, M. A., Photothermal Spectroscopy. In Laser Spectroscopy for Sensing, Baudelet, M., Ed. Woodhead Publ Ltd: Cambridge, 2014; pp 313-361.

30. Selmke, M.; Braun, M.; Cichos, F., Photothermal Single-Particle Microscopy: Detection of a Nanolens. ACS Nano 2012, 6, 2741-9.

31. Selmke, M.; Braun, M.; Cichos, F., Nano-Lens Diffraction around a Single Heated Nano Particle. Opt. Express 2012, 20, 8055-8070.

32. Nedosekin, D. A.; Proskurnin, M. A.; Kononets, M. Y., Model for Continuous-Wave Laser-Induced Thermal Lens Spectrometry of Optically Transparent Surface-Absorbing Solids. Appl. Opt. 2005, 44, 6296-306.

33. El-Kashef, H., Thermo-Optical and Dielectric Constants of Laser Dye Solvents. Review of Scientific Instruments 1998, 69, 1243-1245.

34. Sell, J., Photothermal Investigations of Solids and Fluids; Elsevier Science, 2012.

35. Prod'homme, L., A New Approach to the Thermal Change in the Refractive Index of Glasses. Phys. Chem. Glass 1962, 1, 119-122.

36. Brusnichkin, A. V.; Nedosekin, D. A.; Galanzha, E. I.; Vladimirov, Y. A.; Shevtsova, E. F.; Proskurnin, M. A.; Zharov, V. P., Ultrasensitive Label-Free Photothermal Imaging, Spectral Identification, and Quantification of Cytochrome $\mathrm{C}$ in Mitochondria, Live Cells, and Solutions. J. Biophoton. 2010, 3, 791-806.

37. Moreau, J.; Loriette, V., Confocal Dual-Beam Thermal-Lens Microscope: Model and Experimental Results. Japanese Journal of Applied Physics Part 1-Regular Papers Brief Communications \& Review Papers 2006, 45, 7141-7151.

38. Nedosekin, D. A.; Galanzha, E. I.; Dervishi, E.; Biris, A. S.; Zharov, V. P., SuperResolution Nonlinear Photothermal Microscopy. Small 2014, 10, 135-142.

39. Nedosekin, D. A.; Foster, S.; Nima, Z. A.; Biris, A. S.; Galanzha, E. I.; Zharov, V. P., Photothermal Confocal Multicolor Microscopy of Nanoparticles and Nanodrugs in Live Cells. Drug Metab. Rev. 2015, 47, 346-355. 
Figures for the supporting information

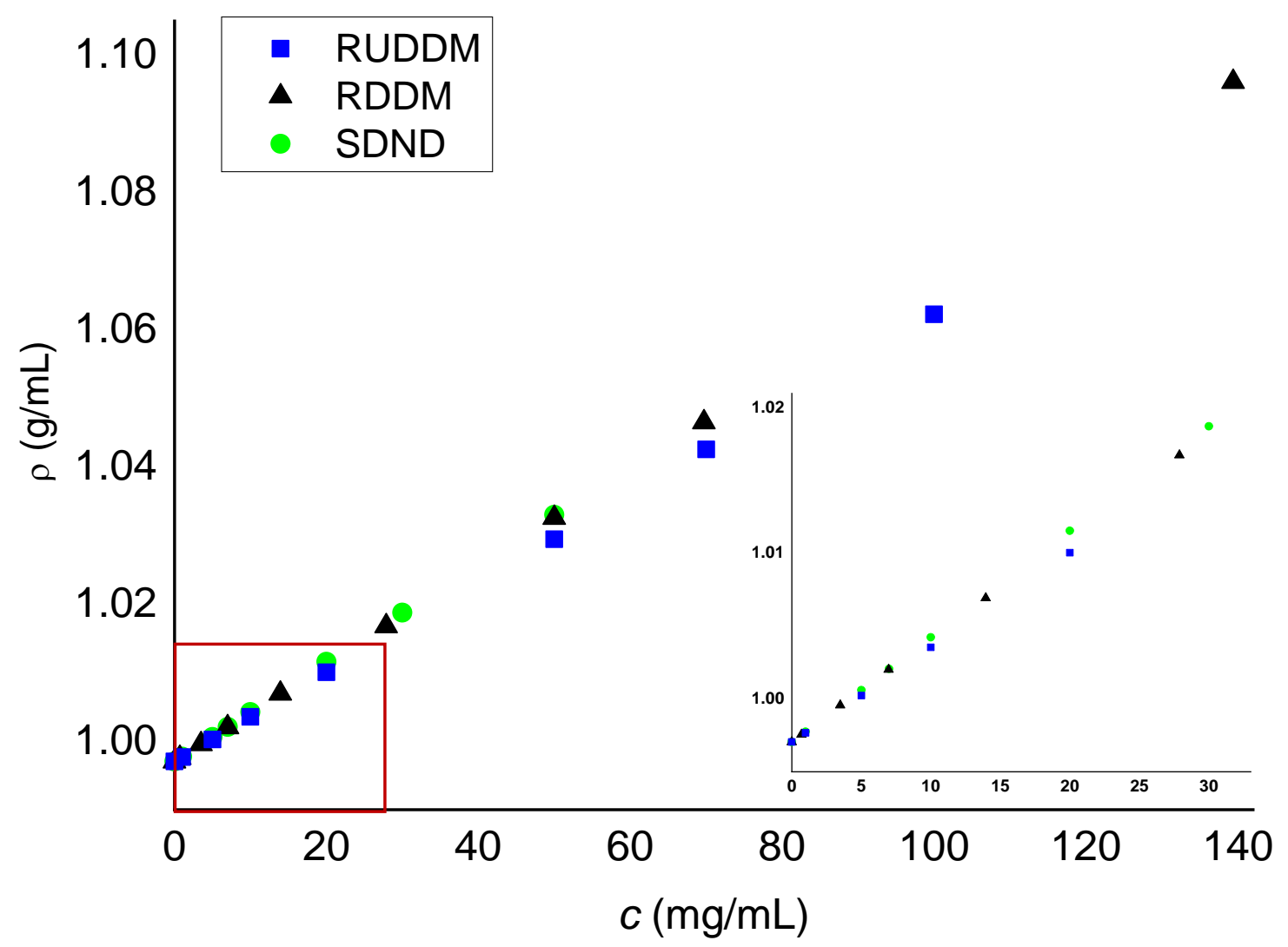

Figure S1. Density of aqueous dispersions of RUDDM, RDDM, and SDND nanodiamonds at $25^{\circ} \mathrm{C}$ as a function of concentration. Inset: the part of low concentrations (framed red) expanded for visibility sake. 

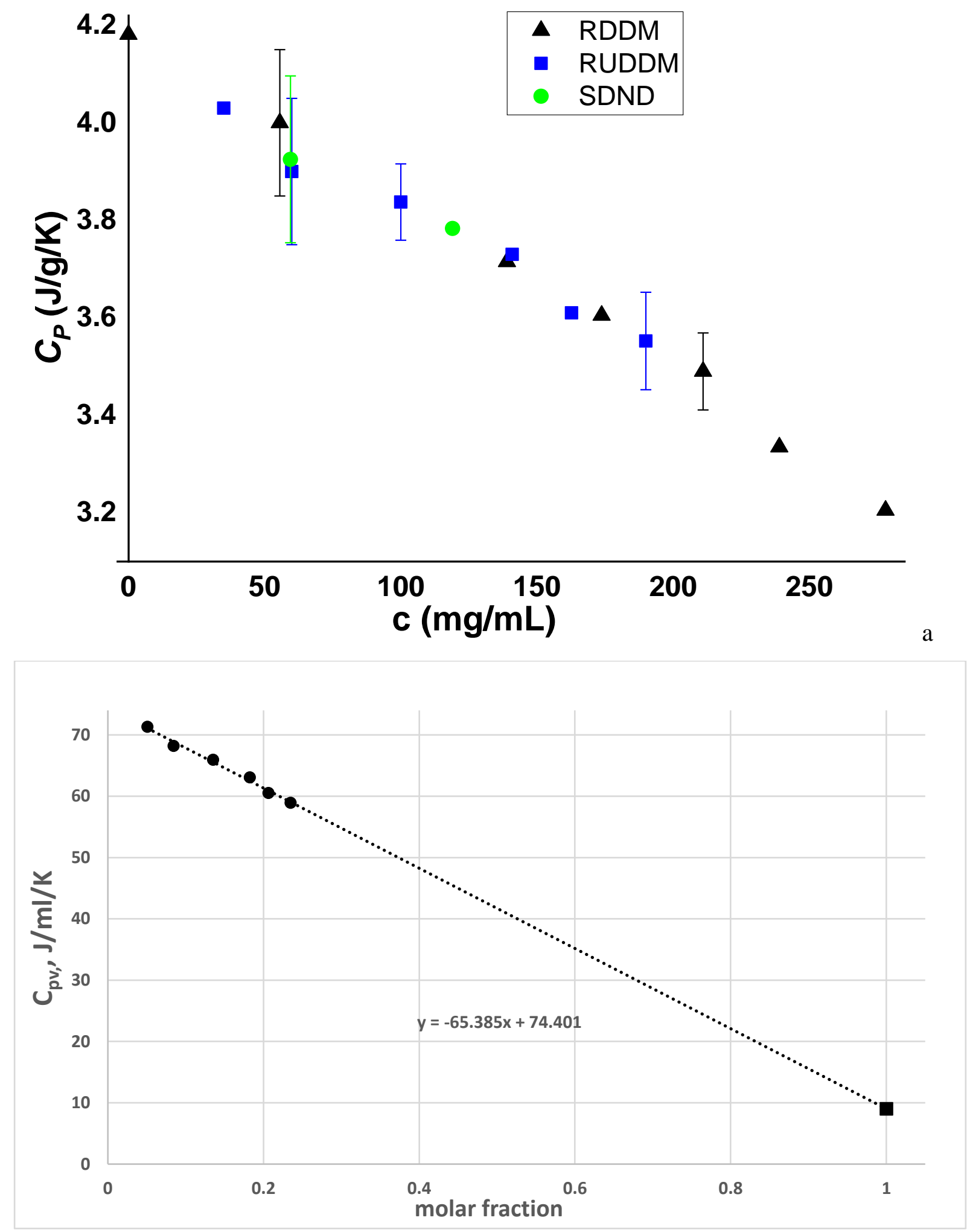

Figure S2. Specific heat of aqueous dispersions of RUDDM, RDDM and SDND nanodiamonds at $25^{\circ} \mathrm{C}$ as a function of concentration (a) and experimental (dots) values of molar specific heat of RUDDM aqueous dispersion on molar fraction of ND; the value of the molar heat capacity of ND solid phase is shown as a square (b). 


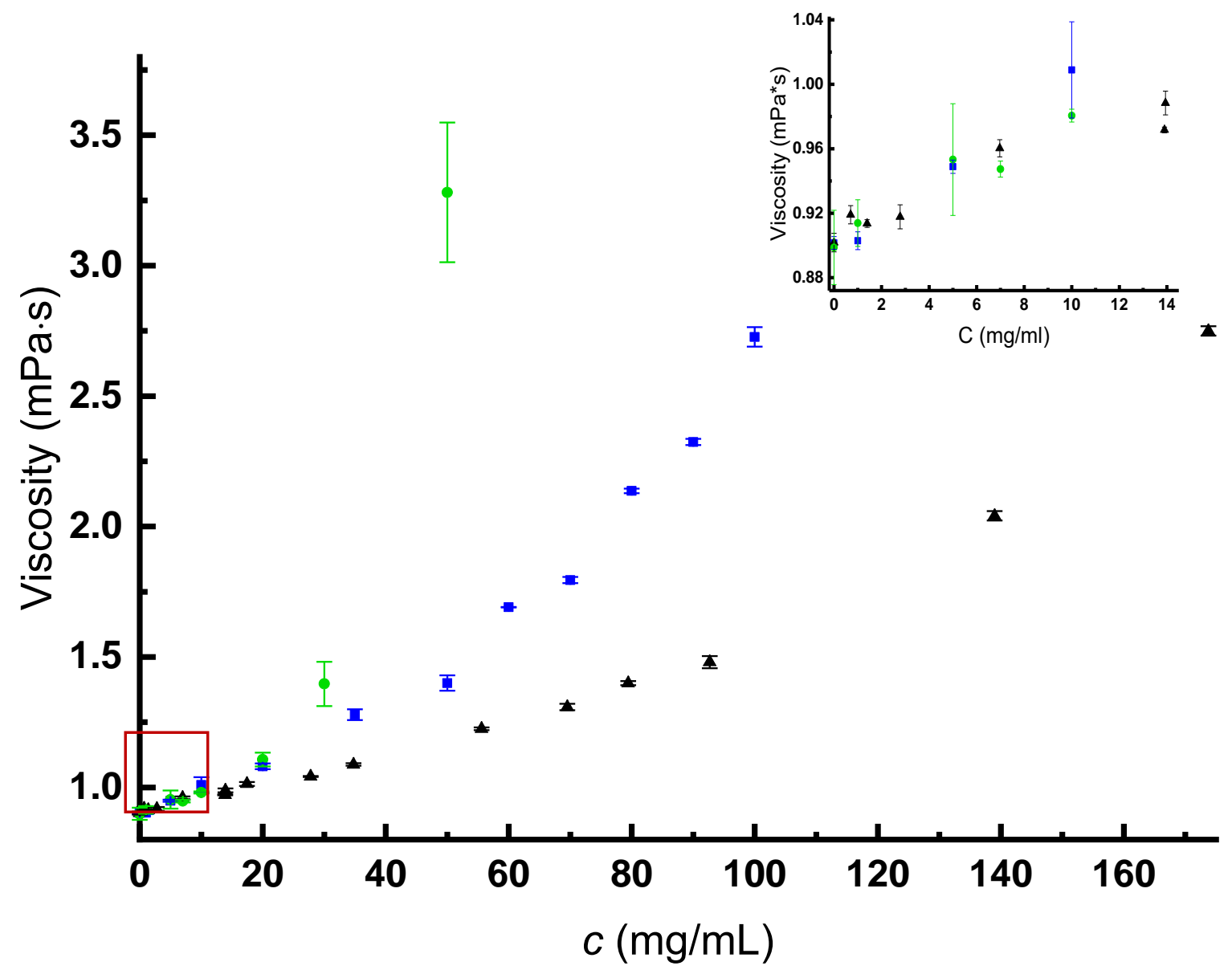

Figure S3. Dynamic viscosity of aqueous dispersions of RUDDM (blue), RDDM (black), and SDND (green) nanodiamonds at $25^{\circ} \mathrm{C}$ as a function of concentration. Inset: the part of low concentrations (framed red) expanded for visibility sake. 


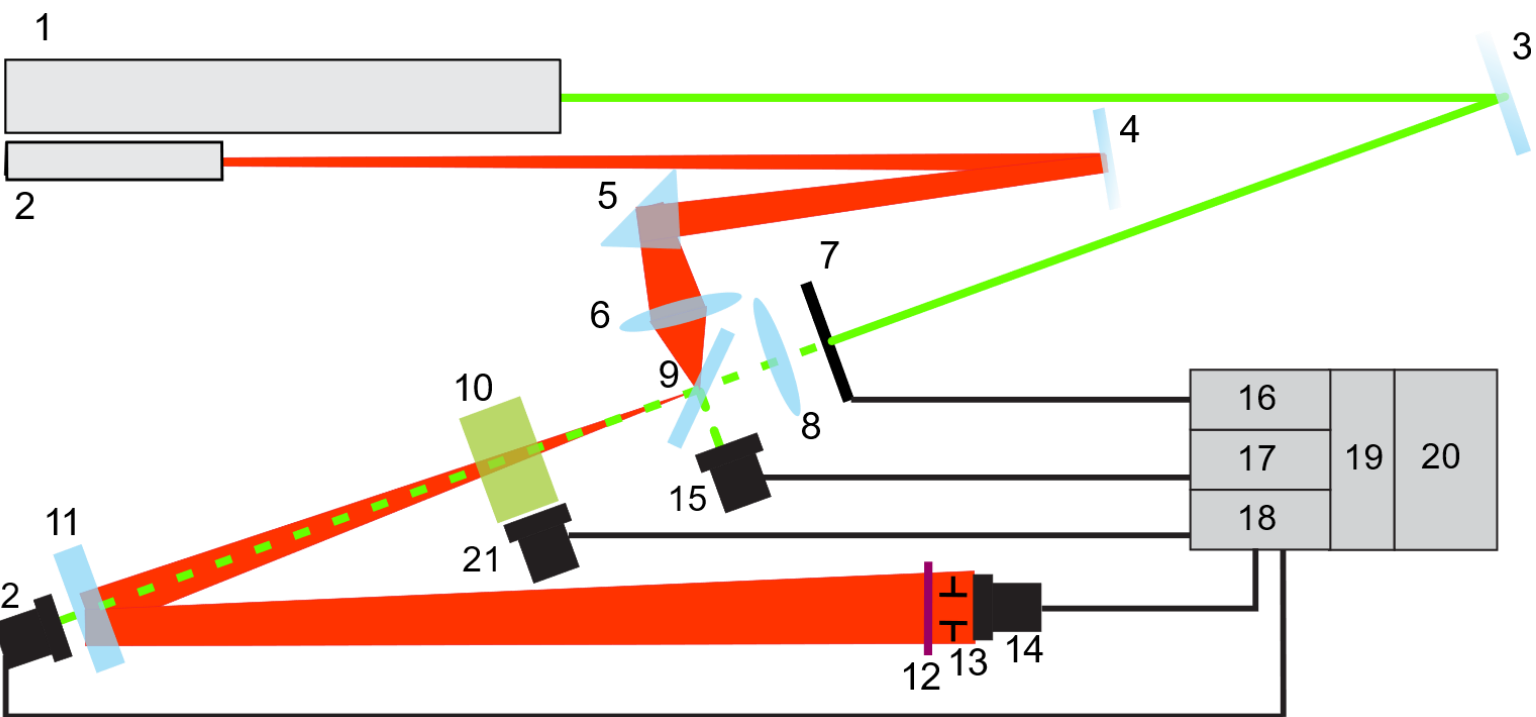

Figure S4. Schematics of the thermal-lens spectrometer. 1 - excitation laser; 2 - probe laser; 3, 4, 9, 11 - diachronic mirrors; 5 - quartz prism; 6 \& 8 - focusing lenses; 7 - chopper; 10 - sample; 12,13 - filter and pinhole; 14,15 - photodiodes; $16,17,18$ - analog amplification units; 19 - DAC-ADC board; 20 - PC. 


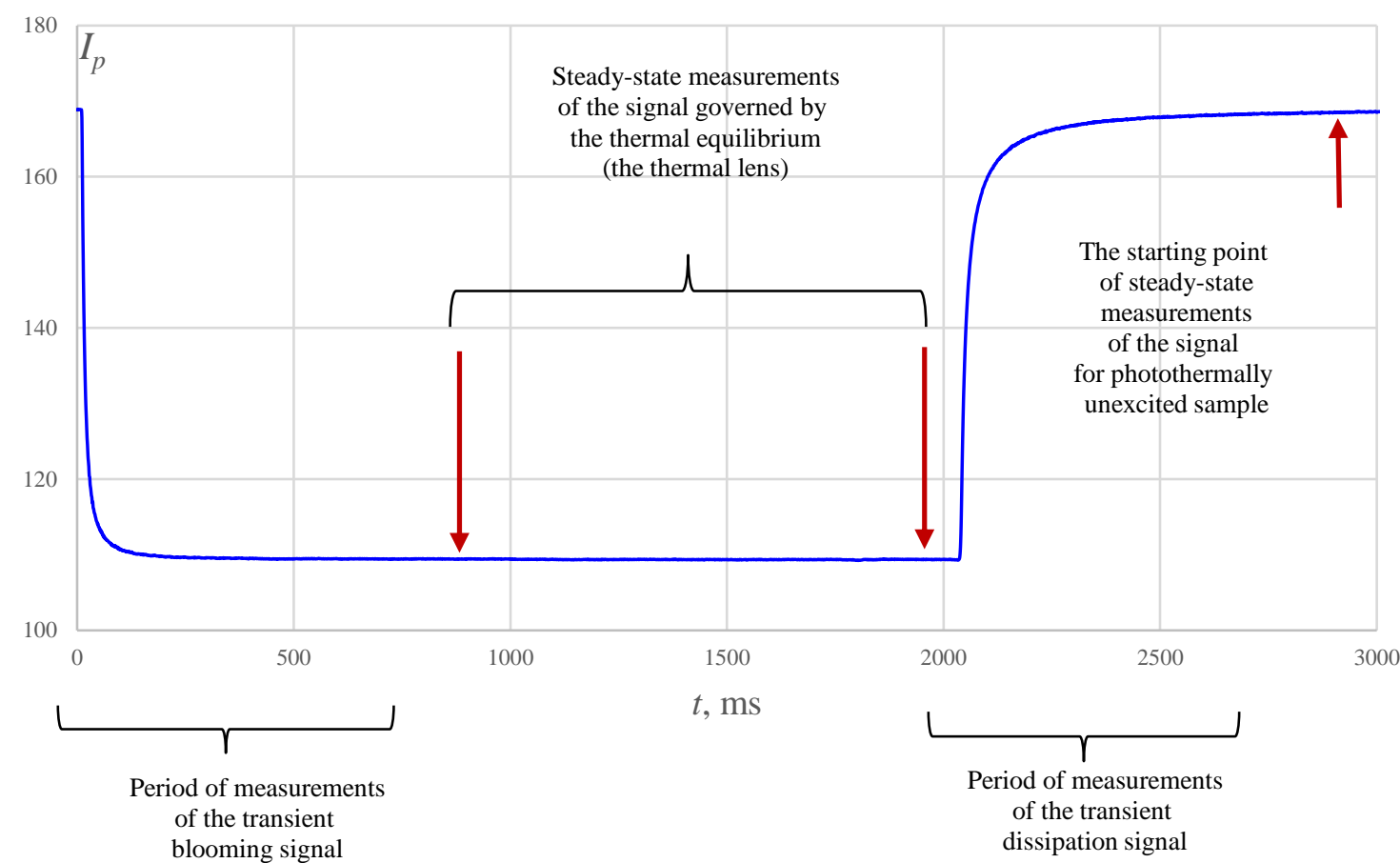

Figure S5. Diagram of transient and steady-state cw thermal-lens measurements in the back-synchronized mode. Reproduced from ${ }^{38}$ 


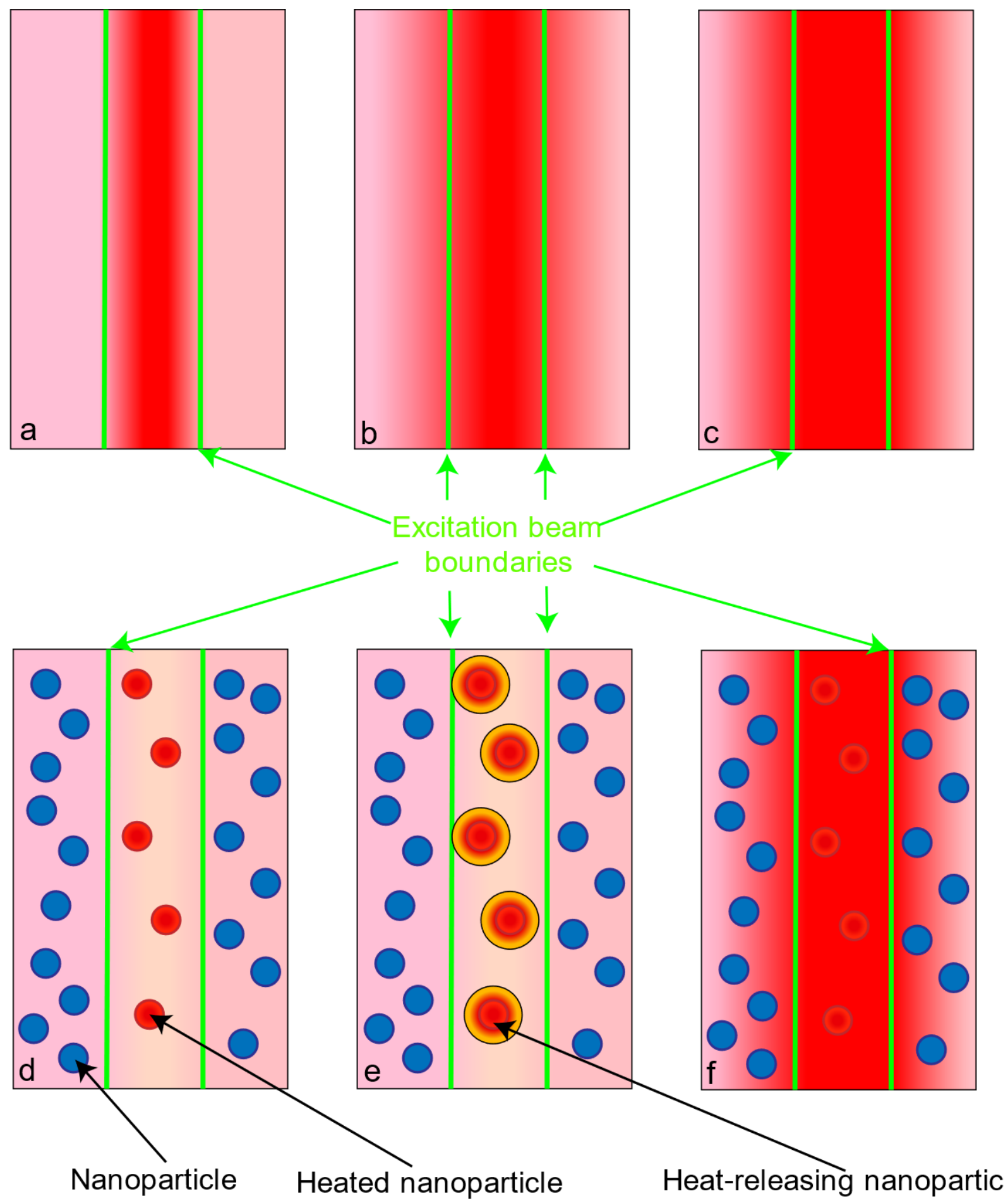

Figure S6. Signal-generation in photothermal lensing of homogenous and disperses systems. 


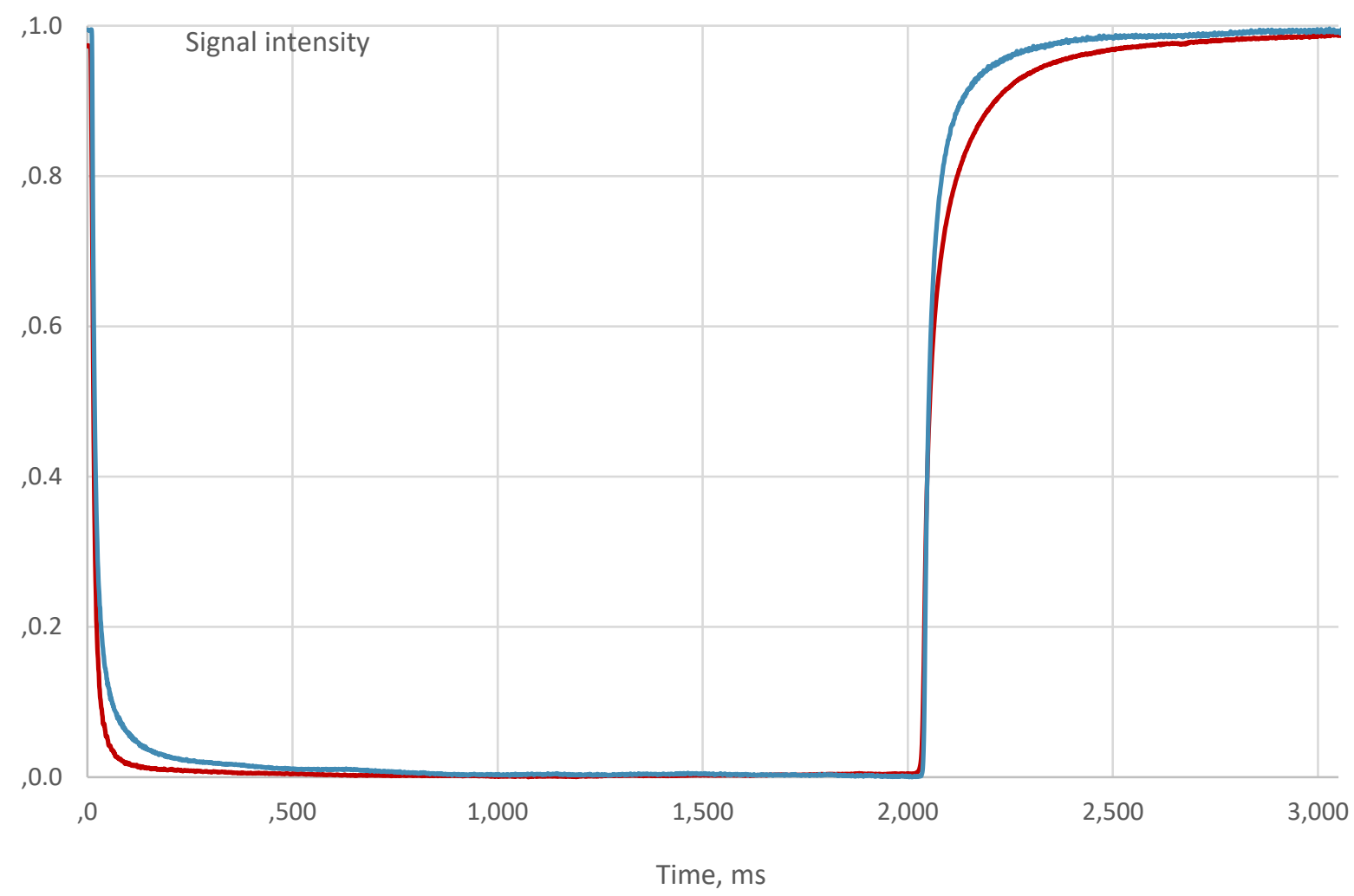

Figure S7. Transient thermal-lens curves for ND (1.0 mg/mL, NanoAmando), red line and a molecular dye (ferroin) in water, blue line; $532 \mathrm{~nm}, 150 \mathrm{~mW}$. 

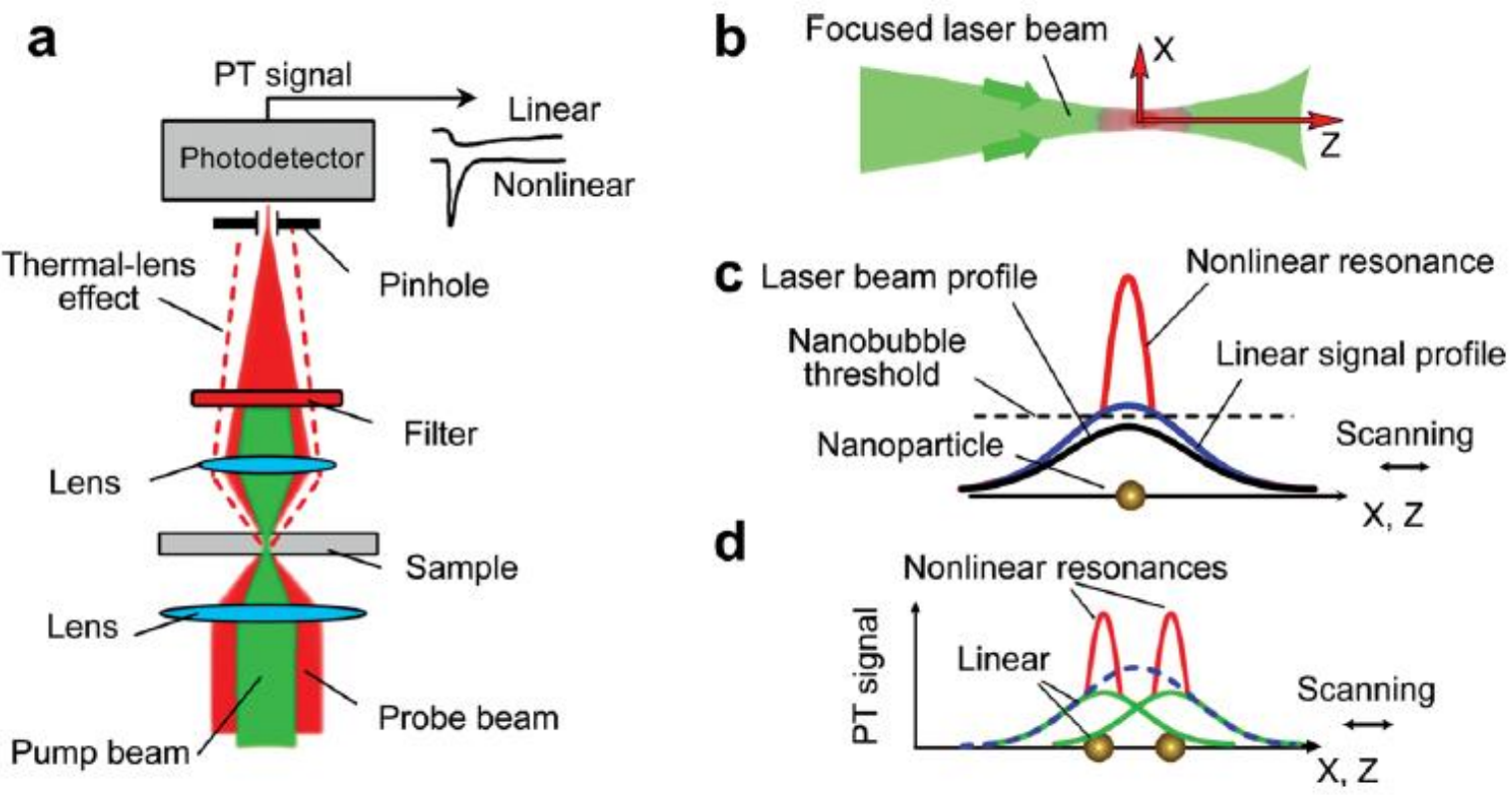

Figure S8. The principles of super-resolution confocal PTM. a) General scheme of a confocal PT microscope. b) Spatial configuration of the focused laser beam. c) Nonlinear PT signal amplification for a nano-object in the center of the laser beam. d) Nonlinear sequential PT spatial resonances during laser scanning from two nano-objects separated by a distance smaller than the size of the diffraction-limited beam spot. The dashed line represents a sum of two linear (green solid lines) PT signals. Reproduced from ${ }^{38}$ 


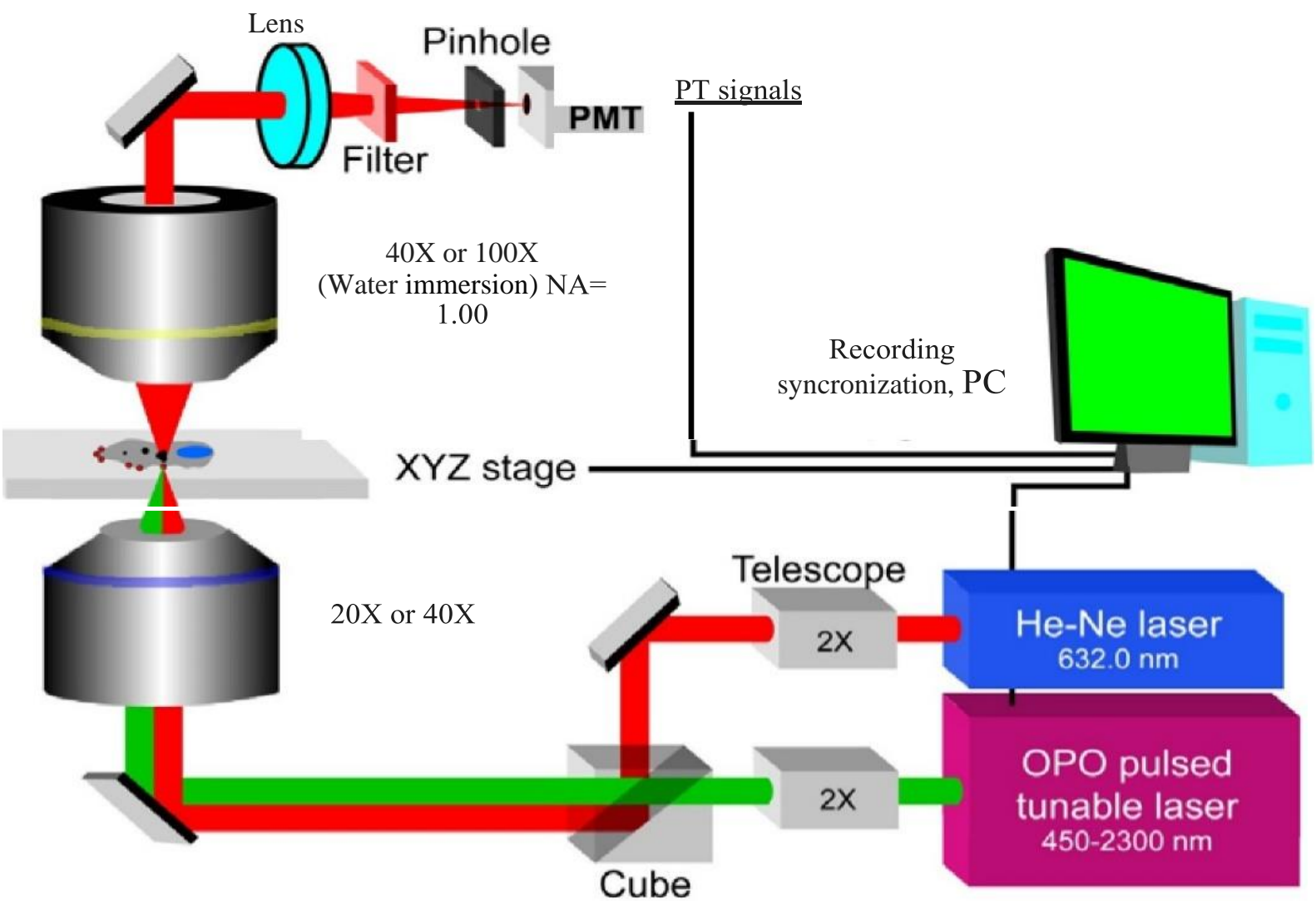

Figure S9. Schematics of PTM (partially reproduced from ${ }^{38}$ ). 


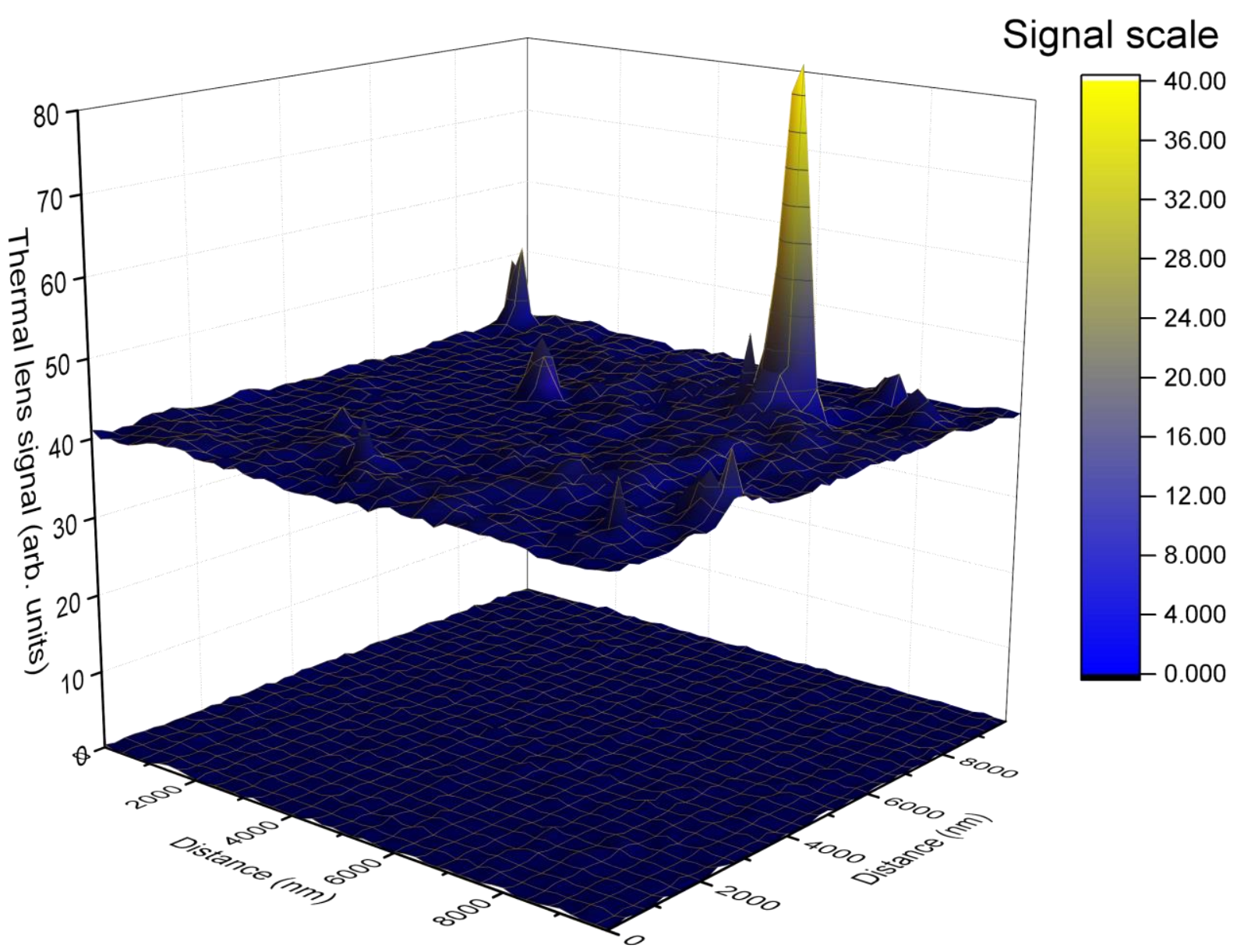

Figure S10. 3D images of photothermal-lens measurements of a blank signal (water) and a NanoAmando nanodiamond dispersion $(4 \mathrm{mg} / \mathrm{mL})$ at the same measurement conditions at $532.0 \mathrm{~nm}$; fluence, $1.25 \mathrm{~J} / \mathrm{cm}^{2}$. The surface plot for NanoAmando is shifted up for the visibility sake. 